\title{
Der Einfluss der Landesverfassungsgerichte auf das Parlamentsrecht der deutschen Bundesländer*
}

\author{
Martina Flick
}

\section{Verfassungsgerichte als Ersatzverfassungsgeber}

Die Rolle von Verfassungsgerichten im politischen Prozess im Allgemeinen und ihr Einfluss auf die Verfassungspolitik im Besonderen haben in den letzten beiden Jahrzehnten vermehrt die Aufmerksamkeit der politikwissenschaftlichen Forschung gefunden. Hierbei wird vielfach eine Justizialisierung der Politik konstatiert, womit ein Machtzuwachs von Gerichten auf Kosten des Parlaments und der Regierung gemeint ist. ${ }^{1}$ Der gerichtliche Einfluss kann sich dabei auf zweierlei Arten äußern: Zum einen können Verfassungsgerichte als Vetospieler fungieren, die gesetzliche Vorhaben der Regierung zu stoppen vermögen ${ }^{2}$; zum anderen nehmen Verfassungsgerichte vielfach die Rolle eines „Ersatzgesetzgebers“ oder gar eines „Ersatzverfassungsgebers“ ein. ${ }^{3}$ Insbesondere im Bereich des Schutzes beziehungsweise des Ausbaus von Grundrechten wird den (nationalen) Verfassungsgerichten erheblicher Einfluss zugeschrieben. Dies gilt nicht nur für das deutsche Bundesverfassungsgericht, das im Wege der verfassungsrichterlichen Rechtsfortbildung dem grundgesetzlichen Grundrechtsteil den Rang einer „objektiven Werteordnung“4 zugeschrieben und daraus sowohl Ausstrahlwirkungen in alle Rechtsbereiche als auch Drittwirkungen, Handlungsaufträge und Schutzpflichten abgeleitet hat. ${ }^{5}$ Ähnliche Entwicklungen sind auch in anderen Staaten zu beobachten. In den USA wurde beispielsweise der Grundsatz der Gleichberechtigung von Mann und Frau durch den Supreme Court hergeleitet, in die Verfassung wurde er bis heute nicht aufgenommen. Auch das Grundrecht der Vereinigungsfreiheit ist in der Verfassung der Vereinigten Staaten nicht enthalten, wird aber vom Supreme Court als Verfassungsgarantie anerkannt. ${ }^{6}$

Aufgrund ihrer schwächeren demokratischen Legitimation ist die Betätigung der Verfassungsgerichte als Ersatzgesetz- beziehungsweise Ersatzverfassungsgeber nicht unproblema-

* Der Beitrag basiert auf Martina Flick, Organstreitverfahren vor den Landesverfassungsgerichten. Eine politikwissenschaftliche Untersuchung, Bern 2011, S. 247 - 276.

1 Vgl. Christine Rothmayr, Towards the Judicialisation of Swiss Politics?, in: West European Politics, 24. Jg. (2001), H. 1, S. 77 - 94, S. 77.

2 So zum Beispiel Nicos C. Alivizatos, Judges as Veto Players, in: Herbert Döring (Hrsg.), Parliaments and Majority Rule in Western Europe, Frankfurt am Main 1995, S. 566 - 589; Uwe Wagschal, Verfassungsgerichte als Vetospieler in der Steuerpolitik, in: Michael Becker / Ruth Zimmerling (Hrsg.), Politik und Recht, PVS-Sonderheft 36, Wiesbaden 2006, S. 559 - 584.

3 Vgl. Alexander von Brünneck, Verfassungsgerichtsbarkeit in den westlichen Demokratien. Ein systematischer Verfassungsvergleich, Baden-Baden 1992, S. 168, S. 170.

4 BVerfGE: Entscheidungen des Bundesverfassungsgerichts 7, 198, S. 205 ff.

5 Vgl. Ernst-Wolfgang Böckenförde, Zur Lage der Grundrechtsdogmatik nach 40 Jahren Grundgesetz, München 1990, S. 31; Thomas Würtenberger, Zur Legitimität des Verfassungsrichterrechts, in: Bernd Guggenberger / ders. (Hrsg.), Hüter der Verfassung oder Lenker der Politik? Das Bundesverfassungsgericht im Widerstreit, Baden-Baden 1998, S. 57 - 80, S. 61.

6 Vgl. Alexander von Brünneck, a.a.O. (Fn. 3), S. 171. 
tisch. ${ }^{7}$ Teilweise werden die von den Verfassungsgerichten entwickelten Rechtssätze zu einem späteren Zeitpunkt in die geschriebene Verfassung aufgenommen, wodurch der demokratietheoretische Mangel ihrer Entstehung formal geheilt wird. Ein Beispiel hierfür stellt die Aufnahme von Art. 3 Abs. 2 Satz 2 ins Grundgesetz dar. ${ }^{8}$ Nach dieser Vorschrift, die 1994 in die Verfassung eingefügt wurde, fördert der Staat die tatsächliche Durchsetzung der Gleichberechtigung von Frauen und Männern und wirkt auf die Beseitigung bestehender Nachteile hin. Vorausgegangen war ein Urteil des Bundesverfassungsgerichts, in dem es um die Verfassungsmäßigkeit einer Vorschrift der Arbeitszeitordnung ging, nach der es verboten war, Arbeiterinnen zur Nachtzeit zu beschäftigen. ${ }^{9}$ Das Gericht sah in der fraglichen Regelung nicht nur einen Verstoß gegen Art. 3 Abs. 3 GG („Niemand darf wegen seines Geschlechts [...] benachteiligt oder bevorzugt werden"), sondern machte zugleich Ausführung darüber, wie Art. 3 Abs. 2 GG („Männer und Frauen sind gleichberechtigt“) zu interpretieren sei. Demnach sollen durch die genannte Vorschrift nicht nur Rechtsnormen beseitigt werden, die Vor- oder Nachteile an Geschlechtsmerkmale anknüpfen. Der über ein Diskriminierungsverbot hinausreichende Regelungsgehalt der Vorschrift bestehe darin, dass er ein Gleichberechtigungsgebot aufstelle, das sich auf die gesellschaftliche Wirklichkeit erstrecke und auf die Angleichung der Lebensverhältnisse von Männern und Frauen gerichtet sei. ${ }^{10}$ Die Verfassungsänderung von 1994 übernimmt also die Rechtsprechung des Bundesverfassungsgerichts in die geschriebene Verfassung.

Hier soll geprüft werden, ob die Rechtsprechung der Verfassungsgerichte in den deutschen Bundesländern die Entwicklung des Parlamentsrechts ähnlich stark beeinflusst hat, insbesondere, ob die Erweiterung von parlamentarischen Minderheitsrechten, die in den letzten Jahren in allen Bundesländern deutlich vorangeschritten ist, auch auf den Einfluss verfassungsgerichtlicher Entscheidungen zurückgeführt werden kann. Der erhebliche Umfang an parlamentsrechtlichen Entscheidungen, den die Landesverfassungsgerichte über die Jahre produziert haben ${ }^{11}$, lässt die Annahme eines vergleichbaren Entwicklungsverlaufs wie bei der Aufnahme beziehungsweise Ausdifferenzierung von Grundrechten plausibel erscheinen. So geht Pascale Cancik davon aus, dass die Abfolge von "Konflikt - Organstreit - Entscheidung - Aufnabme in geschriebenes Recht" ${ }^{12}$ zwar sehr vereinfachend sei, aber dennoch einen typischen Entwicklungspfad im Rahmen des Parlamentsrechts darstelle.

Für die Verfassungsgerichte kann der Ausbau von parlamentarischen Minderheitsrechten längerfristig zu einem Zuwachs an eigenem Einfluss führen. Sofern sie im Rahmen ihrer Rechtsprechungspraxis sehr detaillierte Vorgaben zur Auslegung der einschlägigen Verfassungsbestimmungen machen, kann sich ihre Position an jene der gesetz- beziehungsweise verfassungsgebenden Organe annähern. Zudem ist davon auszugehen, dass die Opposition häufiger Verfassungsstreitigkeiten vor Gericht bringt, wenn das Verfassungsgericht in frü-

7 Vgl. Thomas Würtenberger, a.a.O. (Fn. 5), S. $58 \mathrm{f}$.

8 Vgl. ebenda, S. 62.

9 Vgl. BVerfGE 85, S. 191.

10 Vgl. ebenda, S. $207 \mathrm{f}$.

11 Vgl. Hans-Peter Schneider, Parlamente, Wahlen und Parteien in der Rechtsprechung der Landesverfassungsgerichte, in: Christian Starck / Klaus Stern (Hrsg.), Landesverfassungsgerichtsbarkeit, Baden-Baden 1983, S. 91 - 142, S. 93.

12 Pascale Cancik, Entwicklungen des Parlamentsrechts. Die Bedeutung des verfassungsgerichtlichen Organstreitverfahrens, in: DÖV, 58. Jg. (2005), H. 13, S. 577 - 587, S. 584 (Hervorhebung im Original). 
heren Verfahren ihre verfassungsmäßige Stellung gestärkt hat. Dies gibt den Gerichten wiederum häufiger Gelegenheit, das Verhältnis zwischen Regierung und Opposition mitzugestalten.

Das Untersuchungsmaterial bildet die Rechtsprechung der Landesverfassungsgerichte ${ }^{13}$ in Organstreitverfahren. In dieser Verfahrensart, die in allen Bundesländern vorgesehen ist, entscheiden die Landesverfassungsgerichte über die Auslegung der Verfassung „aus Anlass von Streitigkeiten über den Umfang der Rechte und Pflichten eines obersten Landesorgans oder anderer Beteiligter, die durch die Verfassung oder in der Geschäftsordnung des Landtags oder der Regierung mit eigener Zuständigkeit ausgestattet sind“14. Organstreitigkeiten befassen sich also mit Fragen der Gewaltentrennung und Kompetenzabgrenzung auf höchster Ebene und weisen somit einen direkten Bezug zum Parlamentsrecht auf. Den Verfassungsgerichten bieten sie die Möglichkeit, direkt auf die Rechtsbeziehungen zwischen Regierung und Opposition Einfluss zu nehmen.

\section{Die abhängige Variable: Die Entwicklung des Parlamentsrechts in den Bundesländern}

Die Entwicklung des Parlamentsrechts ist seit etwa 20 Jahren von Änderungen geprägt, durch die die Rechte der parlamentarischen Minderheit gestärkt wurden. Dies gilt beispielsweise für die Festschreibung des Oppositionsprinzips in den meisten Landesverfassungen. Hierdurch soll das in der Praxis bestehende Gegenüber von parlamentarischer Minderheit und Regierungsmehrheit auch normativ abgebildet und die Bedeutung des Oppositionsprinzips unterstrichen werden. ${ }^{15}$ Den Anfang machte Hamburg. Hier wurde bereits 1971 eine entsprechende Vorschrift in die Landesverfassung aufgenommen. Zwischen 1990 und 2000 folgten elf weitere Bundesländer, lediglich in Baden-Württemberg, Hessen, Nordrhein-Westfalen und im Saarland fehlen entsprechende Normierungen. ${ }^{16}$

Eine ähnliche Entwicklung ist bei der Festschreibung von Fraktionsrechten festzustellen. Zwar haben mehrere Landesverfassungen von Anfang an den Fraktionsbegriff verwendet (vgl. Tabelle 1), aber erst seit Beginn der 1990er Jahre wurde deren Rechtsstellung in den Bundesländern durch den Erlass von Fraktionsgesetzen gestärkt. ${ }^{17}$ Auch die meisten Landesverfassungen wurden seither um Ausführungen zur Stellung der Fraktionen oder zu ihren Initiativ- und Mitwirkungsrechten ergänzt. Vorreiter war das Saarland, das den Fraktionen 1979 ein Antragsrecht in Bezug auf die Durchführung von Misstrauensvoten und Normenkontrollverfahren einräumte (Art. 88 und 101 LV Saarland). In den 1990er Jahren folgten die meisten anderen Bundesländer. In diesen neueren Bestimmungen werden die Fraktionen als „selbständige und unabhängige Gliederungen des Landtages“ bezeichnet, die

13 Der Begriff „Landesverfassungsgericht“ dient vorliegend als Sammelbezeichnung für alle Verfassungsgerichte der deutschen Bundesländer unabhängig von deren Eigenbezeichnungen (wie Staatsgerichtshof, Verfassungsgerichtshof) inklusive des Bundesverfassungsgerichts in seiner früheren Eigenschaft als Landesverfassungsgericht von Schleswig-Holstein.

14 Art. 68 Abs. 1 Satz 2 Nr. 1 der Landesverfassung (LV) Baden-Württemberg.

15 Vgl. Pascale Cancik, Die Rezeption neuer Verfassungsregelungen. Ein Beitrag zur „Wirkung“ der Oppositionsregelungen in den Landesverfassungen, in: Jahrbuch des öffentlichen Rechts der Gegenwart, in: Neue Folge, 55. Jg. (2007), S. 151 - 194, S. 153.

16 Vgl. ebenda, S. $154 \mathrm{ff}$.

17 Vgl. ders., a.a.O. (Fn. 12), S. 585. 
mit eigenen Rechten und Pflichten ausgestattet sind und an der parlamentarischen Willensbildung mitwirken. ${ }^{18}$ Häufig wird auch ein Anspruch auf angemessene Ausstattung normiert. Lediglich in Hamburg, Hessen und Nordrhein-Westfalen werden Fraktionen nicht in den Verfassungen erwähnt, in Baden-Württemberg tauchen sie zwar im Verfassungstext auf ${ }^{19}$, es fehlt jedoch an der Zuweisung von eigenen Rechten.

Die parlamentarische Minderheit wurde jedoch nicht nur durch die ausdrückliche Anerkennung des Oppositionsprinzips und der Fraktionsrechte gestärkt. Vielmehr wurden verschiedene Kontroll- und Mitwirkungsrechte, die zuvor nicht vorgesehen waren oder nur von der Mehrheit des Landtags ausgeübt werden konnten, zu Minderheitsrechten umgestaltet. So sehen die Landesverfassungen von Brandenburg, Mecklenburg-Vorpommern und Schleswig-Holstein heute vor, dass das Verlangen einer Minderheit (einem Fünftel bis einem Drittel der Abgeordneten) für die Ausübung des Zitierrechts, also für die Herbeirufung eines Mitglieds der Landesregierung, ausreicht.

Von noch größerer Bedeutung dürfte die Aufnahme von Regelungen sein, die der parlamentarischen Minderheit einen Anspruch auf Vorlage von Regierungsakten einräumen. Hierbei handelt es sich um eines der wenigen Rechte der parlamentarischen Selbstinformation. Die Regierungsseite kann nicht wie beim Zitierrecht oder im Fall von Anfragen den Umfang der Information kontrollieren, da die Aktenvorlage „unverzüglich und vollständig“ erfolgen muss. ${ }^{20}$ Die Herausgabe der Akten darf nur verweigert werden, soweit dem Bekanntwerden des Inhaltes gesetzliche Vorschriften oder das Staatswohl entgegenstehen ${ }^{21}$ oder wenn die Funktionsfähigkeit und Eigenverantwortung der Landesregierung beeinträchtigt werden. ${ }^{22}$ Derzeit sehen fünf Bundesländer entsprechende Minderheitsrechte vor, wobei Hamburg 1971 den Anfang machte. ${ }^{23}$

Eine weitere Informationsquelle wurde den Parlamentariern in den letzten zwei Jahrzehnten in Form von selbständigen Auskunftsrechten zugänglich gemacht. Sie ermöglichen es den Abgeordneten, von der Regierung die Mitteilung von Tatsachen zu verlangen, die sie für ihre Mitwirkung an den parlamentarischen Aufgaben benötigen. Die selbständigen Auskunftsrechte sind insbesondere für die Opposition wichtig, da sie nicht an ein bestimmtes Quorum gebunden sind, sondern von jedem einzelnen Abgeordneten geltend gemacht werden können.

Weitere wichtige Neuerungen im Parlamentsrecht der Länder stellen die Berichtspflichten der Landesregierung dar. Wo sie vorgesehen sind, müssen die Regierungen die Landtage von sich aus über bestimmte Vorgänge unterrichten. Hierzu gehören beispielsweise Bundesratsangelegenheiten, Gesetzesvorhaben, geplante Staatsverträge, Angelegenheiten der Europäischen Union und Grundsatzfragen der Landesplanung. ${ }^{24}$ Durch diese Maß-

18 So zum Beispiel Art. 47 Abs. 2 LV Sachsen-Anhalt.

19 Vgl. Art. 37 LV Baden-Württemberg.

20 Zum Beispiel Art. 53 Abs. 3 LV Sachsen-Anhalt.

21 Vgl. Art. 30 LV Hamburg.

22 Vgl. Art. 23 Abs. 3 LV Schleswig-Holstein.

23 Vgl. Martina Flick, Parlamente und ihre Beziehungen zu den Regierungen, in: Markus Freitag / Adrian Vatter (Hrsg.), Die Demokratien der deutschen Bundesländer. Politische Institutionen im Vergleich, Opladen 2008, S. 161 - 194, S. 181.

24 Vgl. Stefan Storr, Die Pflicht der Landesregierungen zur Unterrichtung der Landtage in parlamentarischen Regierungssystemen, in: Zeitschrift für Gesetzgebung, 20. Jg. (2005), H. 1, S. 45 -65 , S. $54 \mathrm{f}$. 


\begin{tabular}{|c|c|c|c|c|c|c|c|}
\hline \multicolumn{8}{|c|}{$\begin{array}{l}\text { Tabelle 1: Zeitpunkte und Fundstellen der Normierung von parlamentarischen Minderheits- } \\
\text { rechten }{ }^{1}\end{array}$} \\
\hline & $\begin{array}{l}\text { Opposition } \\
\text { in LV }\end{array}$ & \begin{tabular}{|c|} 
Rechte \\
von Fraktio- \\
nen in LV
\end{tabular} & $\begin{array}{l}\text { Zitierrecht } \\
\text { durch } \\
\text { Minderheit }\end{array}$ & $\begin{array}{c}\text { Aktenvorla- } \\
\text { geverlangen } \\
\text { durch } \\
\text { Minderheit }\end{array}$ & $\begin{array}{l}\text { Auskunftsrecht } \\
\text { zugunsten } \\
\text { einzelner } \\
\text { Abgeordneter }\end{array}$ & $\begin{array}{l}\text { Berichts- } \\
\text { pflichten } \\
\text { der } \\
\text { Regierung }\end{array}$ & \begin{tabular}{|c|} 
Gesetzliche \\
Regelung \\
der Untersu- \\
chungsaus- \\
schüsse \\
\end{tabular} \\
\hline BW & - & $--^{2}$ & - & - & - & $\begin{array}{l}1995 \\
\text { Art. } 34 \mathrm{a}\end{array}$ & 1976 \\
\hline BY & $\begin{array}{c}1998 \\
\text { Art. 16a }\end{array}$ & $\begin{array}{c}1998 \\
\text { Art. } 16 \mathrm{a}\end{array}$ & - & - & - & $\begin{array}{c}2003 \\
\text { Art. } 55\end{array}$ & 1970 \\
\hline $\mathrm{BE}$ & $\begin{array}{c}1990 \\
\text { Art. } 38 \\
\end{array}$ & $\begin{array}{c}1991 \\
\text { Art. } 40 \\
\end{array}$ & - & - & - & $\begin{array}{l}1995 \\
\text { Art. } 50\end{array}$ & 1970 \\
\hline BB & $\begin{array}{c}1993 \\
\text { Art. } 55 \\
\end{array}$ & $\begin{array}{c}1993 \\
\text { Art. } 67 \\
\end{array}$ & $\begin{array}{l}1993 \\
\text { Art. } 66\end{array}$ & $\begin{array}{l}1993 \\
\text { Art. } 56\end{array}$ & $\begin{array}{l}1993 \\
\text { Art. } 56\end{array}$ & $\begin{array}{c}1993 \\
\text { Art. } 94 \\
\end{array}$ & 1991 \\
\hline $\mathrm{HB}$ & $\begin{array}{c}1994 \\
\text { Art. } 78\end{array}$ & $1947^{3}$ & - & - & - & $\begin{array}{c}1994 \\
\text { Art. } 79\end{array}$ & 1982 \\
\hline $\mathrm{HH}$ & $\begin{array}{c}1971 \\
\text { Art. } 24\end{array}$ & - & - & $\begin{array}{c}1971 \\
\text { Art. } 32\end{array}$ & - & $\begin{array}{c}1996 \\
\text { Art. } 31 \\
\end{array}$ & 1997 \\
\hline $\mathrm{HE}$ & - & - & - & - & - & - & - \\
\hline MV & $\begin{array}{c}1993 \\
\text { Art. } 26\end{array}$ & $\begin{array}{c}1993 \\
\text { Art. } 25\end{array}$ & $\begin{array}{c}1993 \\
\text { Art. } 38\end{array}$ & - & $\begin{array}{c}1993 \\
\text { Art. } 40\end{array}$ & $\begin{array}{c}1993 \\
\text { Art. } 39\end{array}$ & 2002 \\
\hline NI & $\begin{array}{c}1993 \\
\text { Art. } 19 \\
\end{array}$ & $\begin{array}{r}1993^{4} \\
\text { Art. } 19 \\
\end{array}$ & - & $\begin{array}{c}1993 \\
\text { Art. } 24 \\
\end{array}$ & $\begin{array}{c}1993 \\
\text { Art. } 24 \\
\end{array}$ & $\begin{array}{c}1993 \\
\text { Art. } 25 \\
\end{array}$ & - \\
\hline $\mathrm{NW}$ & - & - & - & - & - & - & 1984 \\
\hline $\mathrm{RP}$ & $\begin{array}{c}2000 \\
\text { Art. } 85 \mathrm{~b}\end{array}$ & $1947^{5}$ & - & - & $\begin{array}{c}2000 \\
\text { Art. } 89 \mathrm{a} \\
\end{array}$ & $\begin{array}{c}2000 \\
\text { Art. } 89 \mathrm{~b}\end{array}$ & 1990 \\
\hline SL & - & $1947^{6}$ & - & - & - & $\begin{array}{c}2001 \\
\text { Art. } 76 \mathrm{a}\end{array}$ & - \\
\hline SN & $\begin{array}{c}1992 \\
\text { Art. } 40\end{array}$ & $\begin{array}{c}1992 \\
\text { Art. } 46 \\
\end{array}$ & - & - & $\begin{array}{c}1992 \\
\text { Art. } 51 \\
\end{array}$ & $\begin{array}{c}1992 \\
\text { Art. } 50 \\
\end{array}$ & 1991 \\
\hline ST & $\begin{array}{c}1992 \\
\text { Art. } 48\end{array}$ & $\begin{array}{c}1992 \\
\text { Art. } 47\end{array}$ & - & $\begin{array}{c}1992 \\
\text { Art. } 53\end{array}$ & $\begin{array}{c}1992 \\
\text { Art. } 53 \\
\end{array}$ & $\begin{array}{c}1992 \\
\text { Art. } 62 \\
\end{array}$ & 1992 \\
\hline $\mathrm{SH}$ & $\begin{array}{c}1990 \\
\text { Art.12 } \\
\end{array}$ & $\begin{array}{c}1990 \\
\text { Art. } 12 \\
\end{array}$ & $\begin{array}{c}1990 \\
\text { Art. } 21 \\
\end{array}$ & $\begin{array}{c}1990 \\
\text { Art. } 23 \\
\end{array}$ & $\begin{array}{c}1990 \\
\text { Art. } 23 \\
\end{array}$ & $\begin{array}{c}1990 \\
\text { Art. } 22 \\
\end{array}$ & 1993 \\
\hline TH & \begin{tabular}{|c|}
1993 \\
Art. 59 \\
\end{tabular} & \begin{tabular}{|c|}
1993 \\
Art. 58 \\
\end{tabular} & - & - & $\begin{array}{c}1995 \\
\text { Art. } 67 \\
\end{array}$ & $\begin{array}{c}1995 \\
\text { Art. } 67\end{array}$ & 1991 \\
\hline \multicolumn{8}{|c|}{$\begin{array}{l}1 \text { Die Artikelangaben beziehen sich auf die jeweiligen Landesverfassungen. } \\
2 \text { Art. } 37 \text { LV Baden-Württemberg erwähnt zwar Fraktionen, spricht ihnen jedoch keine Rechte zu. } \\
3 \text { Nach Art. } 86 \text { Abs. } 1 \text { a.F. und Art. } 105 \text { Abs. } 2 \text { LV Bremen war beziehungsweise ist die Fraktionsstärke } \\
\text { bei der Wahl des Vorstandes sowie bei der Zusammensetzung der Ausschüsse zu berücksichtigen. Eine } \\
\text { ausdrückliche Regelung zur verfassungsmäßigen Stellung der Fraktionen wurde im Jahr } 1994 \text { einge- } \\
\text { fügt (Art. } 77 \text { LV Bremen). } \\
4 \text { Die vorläufige Niedersächsische Verfassung aus dem Jahr } 1951 \text { erwähnte zwar Fraktionen (Art. 14), } \\
\text { sprach ihnen jedoch keine Rechte zu. } \\
5 \text { Fraktionen werden mehrfach in der LV Rheinland-Pfalz von } 1947 \text { als Träger von Rechten erwähnt } \\
\text { (Art. 86, 91, 130). Im Jahr } 1993 \text { wurde eine ausdrückliche Regelung zur verfassungsmäßigen Stellung } \\
\text { der Fraktionen eingefügt (Art. } 85 \text { a LV Rheinland-Pfalz). } \\
6 \text { Nach Art. } 72 \text { Abs. } 2 \text { und Art. } 80 \text { a.F. der LV Saarland ist die Fraktionsstärke bei der Wahl des Land- } \\
\text { tagspräsidiums sowie bei der Zusammensetzung der Ausschüsse zu berücksichtigen. Im Jahr } 1979 \\
\text { wurden weitere Fraktionsrechte eingeführt (Art. } 70 \text { Abs. 2, Art. } 88 \text { Abs. 2, Art. 98, Art. } 101 \text { Abs. } 3 \\
\text { LV Saarland). } \\
\text { Quelle: Eigene Zusammenstellung. }\end{array}$} \\
\hline
\end{tabular}


nahme soll der Informationsvorsprung der Exekutive zumindest teilweise ausgeglichen werden. ${ }^{25}$

Ausdifferenzierungen gab es im Bereich des Rechts der Untersuchungsausschüsse. Sie waren zwar schon immer in den Landesverfassungen vorgesehen, ihre Kompetenzen wurden aber in den meisten Bundesländern erst später im Rahmen von Geschäftsordnungen und eigenständigen Gesetzen über die Einsetzung und das Verfahren von Untersuchungsausschüssen konkretisiert. ${ }^{26}$ Die Entwicklung setzte dabei früher ein als bei den meisten anderen hier genannten Normierungen. So erließen Bayern und Berlin entsprechende Gesetze bereits im Jahr 1970.

\section{Die unabhängige Variable: Die Rechtsprechung der Landesverfassungsgerichte im Parlamentsrecht}

Sofern es zutrifft, dass die verfassungsgerichtliche Rechtsprechung als treibende Kraft für den Ausbau parlamentarischer Minderheitsrechte gewirkt hat, müssen die entsprechenden Entscheidungen notwendigerweise zeitlich vor der Positivierung gelegen haben. Dies wird im Folgenden für die einzelnen Parlamentsrechtsänderungen geprüft.

\subsection{Oppositionsregelung}

Für die erstmalige Aufnahme der Oppositionsregelung, die 1971 in Hamburg erfolgte, lässt sich kein Einfluss der verfassungsgerichtlichen Rechtsprechung feststellen. Das Hamburgische Verfassungsgericht hatte bis zu diesem Zeitpunkt lediglich über ein Organstreitverfahren zu entscheiden gehabt, in dem die Frage nach der verfassungsmäßigen Position der Opposition keine Rolle spielte. ${ }^{27}$ Den Anstoß für die Ergänzung der Verfassung um den Oppositionsartikel ${ }^{28}$ lieferte überraschenderweise der Hamburger Senat, der seinerzeit aus einer Koalition von SPD und FDP bestand. Er brachte den Antrag in die Bürgerschaft ein, die Unvereinbarkeit von Senatorenamt und Abgeordnetenmandat verfassungsrechtlich zu fixieren. Für die FDP hätte dies zur Folge gehabt, dass zwei Listenkandidaten die Mandate ihrer Senatoren übernommen hätten, wodurch sie sich bessere Arbeitsbedingungen im Landesparlament versprach. Die SPD-Fraktion hoffte dagegen, durch die Festlegung des Inkompatibilitätsprinzips eine größere Distanz zum Senat zu gewinnen. ${ }^{29}$ Dem Antrag trat die CDU-Fraktion mit der Begründung entgegen, dass die Unvereinbarkeitsregelung auf „veralteten Vorstellungen von der Gewaltenteilung" beruhe und hierdurch ein „falsches Parlamentsverständnis ${ }^{\text {“ } 30}$ fortgesetzt werde. Schließlich einigten sich die Fraktionen darauf,

25 Vgl. ebenda, S. 45.

26 Vgl. Pascale Cancik, a.a.O. (Fn. 12), S. 585.

27 Vgl. Urteil vom 9. Januar 1963, GVBl. Hamburg 1963, S. 27.

28 Vgl. Art. 24 LV Hamburg.

29 Vgl. Hans-Peter Schneider, Die parlamentarische Opposition im Verfassungsrecht der Bundesrepublik Deutschland, Bd. 1 Grundlagen, Frankfurt am Main 1974, S. 262.

30 Niederschrift über die Sitzung des Ausschusses für Verfassung, Geschäftsordnung und Wahlprüfung vom 26. Oktober 1970, zitiert nach ebenda. 
die von der Regierungsseite gewünschte Inkompatibilitätsregelung ${ }^{31}$ verfassungsrechtlich zu verankern; gleichzeitig sollte jedoch die Normierung eines „falschen Parlamentsverständnisses" dadurch verhindert werden, dass die Verfassung auch Aussagen zur Funktion der Opposition und ihren eigenständigen Aufgaben enthalte. ${ }^{32}$ Die Formulierung, wonach die Opposition „ein wesentlicher Bestandteil der parlamentarischen Demokratie“33 sei, geht dabei auf eine Aussage Erich Ollenhauers im Bundestag aus dem Jahr 1953 zurück. ${ }^{34}$

Erst nach der Normierung der Oppositionsstellung im Rahmen der Landesverfassung von Hamburg setzte in anderen Bundesländern die Auseinandersetzung mit den Aufgaben und Funktionen der Opposition auf verfassungsgerichtlicher Ebene ein. In einer Entscheidung aus dem Jahr 1976 stellt der bayerische Verfassungsgerichtshof fest, dass das Recht zur Bildung und Ausübung einer parlamentarischen Opposition verfassungsmäßig geschützt sei und zu den grundlegenden Prinzipien der freiheitlich demokratischen Grundordnung gehöre. ${ }^{35}$ Ein Jahr später befasste sich der baden-württembergische Staatsgerichtshof mit den Befugnissen der Opposition im Zusammenhang mit der Einsetzung von Untersuchungsausschüssen. ${ }^{36}$ Dabei wurde betont, dass die Kontrollfunktion der Untersuchungsausschüsse sich nicht nur auf das Verhältnis zwischen dem Parlament als Ganzem und der Exekutive beziehe, sondern auch auf das Verhältnis zwischen „der Opposition und der Parlamentsmehrheit“ ${ }^{\text {37. }}$.

\subsection{Fraktionsrechte}

Der Status der Fraktionen war schon früh Gegenstand von Verfahren. Vor dem Staatsgerichtshof von Niedersachsen ging es beispielsweise um die Frage, welcher Fraktion das Recht zustehe, den Posten des Landtagspräsidenten zu besetzen, wenn es im Laufe der Wahlperiode zu Veränderungen der Mehrheitsverhältnisse im Landtag komme. ${ }^{38}$ Dies war im konkreten Fall durch den Übertritt von 16 Abgeordneten der Deutschen Partei zur CDU-Fraktion erfolgt. Der Staatsgerichtshof verwarf den von der CDU-Fraktion gestellten Antrag als unzulässig, da die auszulegende Vorschrift lediglich in der Geschäftsordnung des Landtags, nicht aber in der Landesverfassung verankert sei. Auch in einem weiteren Verfahren, das kurze Zeit später von den verbliebenen Abgeordneten der Deutschen Partei initiiert wurde, baute der Staatsgerichtshof die Rechtsposition der Fraktionen nicht aus. ${ }^{39}$ Zwar gingen die Richter in diesem Fall von der Parteifähigkeit von Fraktionen in Organstreitverfahren aus, da sie durch die Geschäftsordnung des Landtags mit eigenen Zuständigkeiten ausgestattet seien. Durch den Übertritt von DP-Abgeordneten zur CDU habe die antragstellende Deutsche Partei jedoch den Fraktionsstatus verloren. Angesichts der Aufgaben, die

31 Vgl. Art. 39 LV Hamburg.

32 Vgl. Hans-Peter Schneider, a.a.O. (Fn. 29), S. 263.

33 Art. 24 Abs. 1 LV Hamburg.

34 Vgl. Hans-Peter Schneider, a.a.O. (Fn. 29), S. 261.

35 Vgl. Urteil vom 30. April 1976, BayVerfGHE 29, S. 62 (S. 91), zitiert nach Hans-Peter Schneider, a.a.O. (Fn. 11), S. 129.

36 Vgl. Urteil vom 16. April 1977, ESVGH 27, S. 1.

37 Hans-Peter Schneider, a.a.O. (Fn. 11), S. 130.

38 Vgl. Urteil vom 13. August 1962, NdsStGHE 1, S. 83.

39 Vgl. Urteil vom 19. Januar 1963, NdsStGHE 1, S. 90. 
eine Fraktion zu erfüllen habe, sehe die Geschäftsordnung des Landtags eine gewisse Mindeststärke als erforderlich an, die durch die Vereinigung von lediglich zwei verbliebenen Abgeordneten nicht mehr gegeben sei. ${ }^{40}$ Die Entscheidung des Niedersächsischen Staatsgerichtshofs befasste sich also nur insoweit mit dem Begriff der Fraktion, als zu prüfen war, ob die Antragsberechtigung der beiden Abgeordneten als Fraktion im Sinne der Geschäftsordnung gegeben war. Eine Auseinandersetzung mit dem Status von Fraktionen fand nicht statt.

Eine Aufwertung erfuhren die Fraktionen durch eine Entscheidung des Bremischen Staatsgerichtshofs. Dieser hatte über die Rechtsmäßigkeit eines Fraktionsausschlusses zu entscheiden. ${ }^{41}$ Der betroffene Abgeordnete vertrat dabei die Ansicht, dass ein solcher Schritt nicht ohne Einschaltung der Wahlgremien der Partei möglich sei. Der Staatsgerichtshof entschied, dass Fraktionen den Rang von ständigen, mit eigenen Rechten ausgestatteten Gliederungen der Bürgerschaft einnähmen. Zwar hätten sie eine Doppelstellung, da sie zugleich im Parlament wie auch mittelbar als Repräsentant der jeweiligen Partei fungierten. Den Parteien stünden jedoch keine Einwirkungsrechte auf die den Fraktionen obliegenden Aufgaben zu, zu denen insbesondere die Fraktionsbildung gehöre. ${ }^{42}$ Der erfolgte Fraktionsausschluss wurde daher als verfassungsmäßig angesehen.

Eine verfassungsprozessuale Anerkennung erfuhren die Fraktionen durch ein Urteil des Bayerischen Verfassungsgerichtshofs ${ }^{43}$, obwohl die Landesverfassung zum damaligen Zeitpunkt die Fraktionen nicht ausdrücklich vorsah. In der Sache ging es hierbei um die Frage, ob Teile des Rundfunkänderungsgesetzes vom 1. März 1972 mit Art. 3 LV Bayern (Rechtsstaatsprinzip) vereinbar seien. Gegen das Gesetz hatte der Bayerische Senat Einwendungen erhoben, was eine erneute Beratung und Abstimmung im Landtag nach sich zog (Art. 41 Abs. 2 a.F. LV Bayern). Hierbei stimmte die Mehrheit der Abgeordneten gegen die Einwendungen des Senats. Nach der Ausfertigung und Veröffentlichung des Gesetzes rief die Landtagsfraktion der SPD den Verfassungsgerichtshof an, Antragsgegnerin war die CSUFraktion. Das Gericht entschied, dass Fraktionen als Streitteile in Verfassungsstreitigkeiten nach Art. 75 Abs. 3 LV Bayern auftreten können ${ }^{44}$, sofern sie, wie hier geschehen, die überwiegende Mehrheit der mit gegenteiliger Auffassung sich gegenüberstehenden Landtagsabgeordneten in sich vereinigen. ${ }^{45}$

Wenige Jahre später entschied dasselbe Gericht in einem Grundsatzurteil über die Zulässigkeit der Festlegung einer bestimmten Fraktionsmindestgröße ${ }^{46}$ Bei der Landtagswahl 1974 hatte die FDP mehr als fünf Prozent der Wählerstimmen erhalten und war mit acht Abgeordneten in den Landtag eingezogen. Kurz vor der Wahl war durch eine Änderung der Geschäftsordnung die Mindestgröße zur Bildung einer Fraktion von fünf auf zehn Abgeordnete hinaufgesetzt worden. Die acht FDP-Abgeordneten wollten gegenüber dem Landtag ihre Anerkennung als Fraktion erreichen. Dies wurde ihnen von der Parlamentsmehrheit verweigert, was die Einleitung eines Organstreitverfahrens zur Folge hatte. Zur

40 Vgl. ebenda, S. 99.

41 Vgl. Entscheidung vom 13. Juli 1970, BremStGHE 1970/1976, 19.

42 Vgl. Hans-Peter Schneider, a.a.O. (Fn. 11), S. 109.

43 Vgl. Entscheidung vom 27. Juli 1972, BayVerfGHE 25, S. 97.

44 Es handelt sich hierbei also nicht um ein Organstreitverfahren.

45 Vgl. BayVerfGHE 25, S. 97 (S. 108).

46 Vgl. Entscheidung vom 30. April 1976, BayVerfGHE 29, S. 62. 
Begründung ihres Antrags verwiesen die FDP-Abgeordneten insbesondere darauf, dass die Festlegung einer Fraktionsmindestgröße verfassungswidrig sei, sofern die Fraktion von Abgeordneten einer Partei gebildet werden soll, die bei der Wahl mindestens fünf Prozent der gültigen Stimmen auf sich vereinigen konnte.

Der Verfassungsgerichtshof folgte dieser Argumentation nicht. Als Gliederungen des Parlaments unterlägen die Fraktionen dem Parlamentsrecht und damit auch der Geschäftsordnung des Landtags. Bei Erlass der Geschäftsordnung sei das Parlament weitgehend autonom, allerdings seien die verfassungsmäßigen Schranken zu beachten. Hierzu zählten das Willkürverbot, der Grundsatz der Wahlgleichheit, die Rechte aus dem freien Mandat der Abgeordneten, das aus dem Rechtsstaatsprinzip abgeleitete Übermaßverbot, die Oppositionsfreiheit sowie der Minderheitsschutz. ${ }^{47}$ Die angegriffene Geschäftsordnungsregelung überschreite diese Schranken nicht, sondern orientiere sich an sachbezogenen Kriterien. Die Funktionsfähigkeit des Parlaments setze eine gewisse Arbeitsteilung und damit auch Einschränkungen der Eigenständigkeit der Abgeordneten voraus. Im Interesse der Funktionsfähigkeit des Parlaments müsse dies in Kauf genommen werden. ${ }^{48}$ Zudem könne durch die Zuerkennung des Fraktionsstatus an kleine, weniger bedeutende parlamentarische Gruppen eine unangemessene Bevorzugung gegenüber großen Fraktionen eintreten. ${ }^{49}$

Wenn auch der Antrag der FDP-Abgeordneten in der Sache keinen Erfolg hatte, führte das Urteil zu einer Anerkennung der Bedeutung von Fraktionen. Obwohl sie in der Bayerischen Verfassung zum damaligen Zeitpunkt nicht erwähnt wurden, sprach ihnen der Verfassungsgerichtshof in Anlehnung an entsprechende Entscheidungen des Bundesverfassungsgerichts den Rang von notwendigen Einrichtungen des Verfassungslebens und von mit eigenen Rechten ausgestatteten Teilen eines Staatsorgans zu. Aufgrund ihrer Bedeutung in der Verfassungswirklichkeit sei ihnen auch die Parteifähigkeit in Organstreitverfahren zuzugestehen, obwohl die Bayerische Verfassung keine dem Art. 93 Abs. 1 Nr. 1 GG entsprechende Vorschrift enthalte, nach der es für die Parteifähigkeit ausreicht, dass der Beteiligte in der Geschäftsordnung eines obersten Bundesorgans mit eigenen Rechten ausgestattet ist. ${ }^{50}$ Darüber hinaus seien auch parlamentarische Gruppen parteifähig, jedenfalls dann, wenn sie im Rahmen des Organstreits gerade die Zuerkennung des Fraktionsstatus geltend machen.

Zusammenfassend lässt sich festhalten, dass die Rechtsprechung der Landesverfassungsgerichte kaum den Anstoß zur Festschreibung von Fraktionsrechten in den Landesverfassungen gegeben haben dürfte. Allerdings enthalten die erwähnten Entscheidungen der Gerichte in Bremen und Bayern inhaltliche Auseinandersetzungen mit den Rechten von Fraktionen, durch die deren Position gefestigt beziehungsweise ausgebaut wurden. ${ }^{51}$ Dies war insbesondere in Bayern bedeutsam, da die dortige Verfassung zum Zeitpunkt der Entscheidung keine Aussagen zu Rechten und Pflichten der Fraktionen enthielt. Zudem weitete der Verfassungsgerichtshof die Parteifähigkeit in Organstreitverfahren entgegen dem Wortlaut der Verfassung auf Fraktionen und parlamentarische Gruppen aus.

47 Vgl. ebenda S. 88 ff.; Hans-Peter Schneider, a.a.O. (Fn. 11), S. 110 f.

48 Vgl. BayVerfGHE 29, S. 62 (S. 90).

49 Vgl. BayVerfGHE 29, S. 62 (S. 92).

50 Vgl. ebenda S. 81.

51 Vgl. BremStGHE 1970/1976, 19; BayVerfGHE 29, S. 62. 


\subsection{Zitierrecht}

Das Zitierrecht war, soweit festgestellt werden konnte, noch nie Gegenstand von Verfassungsstreitigkeiten auf Landesebene, so dass die Annahme, die Rechtsprechung der Landesverfassungsgerichte habe insoweit den Anstoß für die Normierung des Minderheitsrechts in der Verfassung gegeben, mit Sicherheit verneint werden kann.

\subsection{Vorlage von Regierungsakten}

Ähnliches gilt für den Minderheitsanspruch auf Vorlage von Regierungsakten, der erstmals im Jahr 1971 in eine Landesverfassung aufgenommen wurde. Vor diesem Zeitpunkt gab es keine einschlägigen Verfassungsgerichtsentscheidungen. Vielmehr ist sogar ein entgegengesetzter Wirkungszusammenhang ersichtlich: Die Rechtsprechung in Organstreitverfahren sorgte nicht für die Positivierung des Anspruchs, sondern die Aufnahme des Aktenvorlageanspruchs in fünf Landesverfassungen führte dazu, dass seine Geltendmachung mehrere Verfassungsstreitigkeiten zwischen Regierung und Opposition nach sich zog. ${ }^{52}$ In diesen Fällen ging es um die Konkretisierung von Inhalt und Grenzen des Aktenvorlageanspruchs. Streitig wurde dabei beispielsweise, ob die Landesregierung die Herausgabe der begehrten Akten mit der Begründung verweigern durfte, dass hierdurch die Funktionsfähigkeit und Eigenverantwortung der Landesregierung wesentlich beeinträchtigt würden. Entsprechende Einschränkungen des Vorlageanspruchs sind in den Landesverfassungen von Niedersachsen, Sachsen-Anhalt und Schleswig-Holstein vorgesehen. Ein weiterer Einwand, der hin und wieder von Landesregierungen gegen das Aktenvorlageverlangen vorgebracht wurde, betraf datenschutzrechtliche Interessen, die durch die Akteneinsicht gefährdet seien.

\subsection{Individuelle Auskunftsansprüche}

Auch für die Festschreibung individueller Auskunftsrechte zugunsten der Abgeordneten kann die verfassungsgerichtliche Rechtsprechung als treibende Kraft ausgeschlossen werden. Solche Rechte wurden vor allem zwischen 1990 und 1993 in mehreren Verfassungen normiert, 1995 und 2000 folgten Thüringen und Rheinland-Pfalz (vgl. Tabelle 1). Die erste verfassungsgerichtliche Entscheidung (einschließlich der Rechtsprechung des Bundesverfassungsgerichts), die einen Anspruch auf Auskunft herleitete, erging aber erst im Oktober 1993 durch den Verfassungsgerichtshof von Nordrhein-Westfalen ${ }^{53}$, also zu einem Zeitpunkt, als bereits in sechs Bundesländern entsprechende Verfassungsregelungen in Kraft waren. $\mathrm{Zu}$ einer Festschreibung in der nordrhein-westfälischen Landesverfassung ist es allerdings bislang nicht gekommen. Zwar wurde die Positivierung des Anspruchs im Rahmen

52 Vgl. zum Beispiel die Entscheidungen des Hamburgischen Verfassungsgerichts vom 6. Juli 1973, JVBl. Hamburg 1973, S. 282, S. 286; Entscheidung des Verfassungsgerichts Brandenburg vom 20. Juni 1996, LVerfGE 4, S. 179; Entscheidung des Niedersächsischen Staatsgerichtshof vom 15. Mai 1996, NdsStGHE 3, S. 252.

53 Vgl. OVGE MüLü 43, S. 274. 
eines Gesetzentwurfs geprüft ${ }^{54}$, den die FDP-Fraktion im März 2002 eingebracht hatte und der darüber hinaus weitere Regelungen anstrebte, die zu einer Stärkung der parlamentarischen Minderheitsrechte (Unterrichtungspflicht der Landesregierung, Aktenvorlage, Aufnahme einer Oppositionsregelung in die Landesverfassung) beitragen sollten. Der Entwurf wurde im Hauptausschuss des Landtags beraten, infolge des Ablaufs der Wahlperiode wurden die Beratungen aber nicht zum Abschluss gebracht. Ein vergleichbarer Gesetzentwurf wurde im März 2006 durch die Fraktionen von SPD und Grünen eingebracht ${ }^{55}$, auch er verfiel mit Ablauf der Wahlperiode.

Im Übrigen lässt sich für das Verhältnis zwischen Rechtsprechung und Positivierung von Parlamentsrecht in Bezug auf die selbständigen Auskunftsrechte der Abgeordneten derselbe Wirkungszusammenhang erkennen, der auch schon beim Anspruch auf Vorlage von Regierungsakten festgestellt wurde: Die verfassungsgerichtliche Rechtsprechung gab nicht den Anstoß für die Normierung des Rechts, vielmehr hatte die Aufnahme des Rechts in die Verfassung zur Folge, dass zahlreiche Organstreitverfahren durchgeführt wurden, die sich mit der Frage beschäftigten, ob die Landesregierung die entsprechenden Auskünfte „nach bestem Wissen unverzüglich und vollständig "56 gegeben habe. Beispiele bilden die Verfahren in Mecklenburg-Vorpommern, in Niedersachsen und eine Reihe von Verfahren vor dem Sächsischen Verfassungsgerichtshof. ${ }^{57}$

\subsection{Berichtspflichten der Regierungen}

Auch die neu eingeführten Berichtspflichten der Regierungen, die seit 1990 in mehrere Landesverfassungen aufgenommen wurden, gehen nicht auf die Rechtsprechung der Landesverfassungsgerichte zurück. Entscheidungen, die eine solche Berichtspflicht für notwendig oder wünschenswert erachten, sind nicht zu finden. Anders als beim Minderheitsanspruch auf Aktenvorlage kam es auch nach der Normierung nicht zu Verfassungsstreitigkeiten, die sich hierauf bezogen.

\subsection{Untersuchungsausschüsse}

Ganz anders stellt sich die Lage hinsichtlich der Untersuchungsausschüsse dar. ${ }^{58}$ Hierzu sind zahlreiche Entscheidungen der Landesverfassungsgerichte ergangen, die über jene des Bundesverfassungsgerichts sowohl zahlenmäßig als auch hinsichtlich ihrer Bedeutung in „eklatantem $\mathrm{Maße}{ }^{\text {"59 }}$ hinausgehen und verschiedene Dimensionen des parlamentarischen Untersuchungsrechts zum Inhalt haben. Ein Teil der Entscheidungen beschäftigt sich mit den Anforderungen, die an den Einsetzungsantrag zu stellen sind. Dieser muss „bestimmt“

54 Vgl. Drucksache 13/2393.

55 Vgl. Landtagsdrucksache 14/1541.

56 Zum Beispiel Art. 51 Abs. 1 LV Sachsen; Art. 23 Abs. 1 LV Schleswig-Holstein.

57 Vgl. Mecklenburg-Vorpommern: Urteil vom 19. Dezember 2002, LVerfGE 13, S. 284; Niedersachsen: Beschluss vom 25. November 1997, NdsStGHE 3, S. 322; Sachsen: zum Beispiel Urteil vom 16. April 1998, LVerfGE 8, S. 282.

58 Vgl. Hans-Peter Schneider, a.a.O. (Fn. 11), S. 103 ff.

59 Ebenda, S. 103. 
sein, das heißt der Untersuchungsgegenstand muss hinreichend klar bezeichnet sein. ${ }^{60}$ Inhaltlich muss er sich auf Materien beziehen, die in den Wirkungsbereich des Parlaments fallen. ${ }^{61}$ Die Aufklärung muss zudem im öffentlichen Interesse liegen. ${ }^{62}$ Andere Entscheidungen enthalten Aussagen zum Status der Untersuchungsausschüsse. So bezeichnete der Hessische Staatsgerichtshof im Jahr 1966 Untersuchungsausschüsse als „Hilfsorgane des Landtags"63.

Eng mit der Frage nach dem Status von Untersuchungsausschüssen verbunden ist der Problemkreis der Minderheitsrechte. Da alle Landesverfassungen das Recht, die Einsetzung eines Untersuchungsausschusses zu verlangen, einer qualifizierten Minderheit zusprechen, kann diese auch das Untersuchungsthema bestimmen. Die Landtagsmehrheit darf den Inhalt und Umfang der Untersuchungstätigkeit nicht nachträglich durch Zusatzfragen erweitern oder verändern. Bestehen Zweifel an der Verfassungsmäßigkeit des Minderheitsantrags, so kann der Landtag den Einsetzungsbeschluss auf die verfassungsmäßigen Teile beschränken. Dabei darf das Thema aber nicht derart verkürzt werden, dass das Recht der Minderheit wesentlich beeinträchtigt wird. Zudem darf das Untersuchungsziel als solches nicht geändert oder gar in sein Gegenteil verkehrt werden. ${ }^{64}$

Zahlreiche Entscheidungen setzen sich mit den Modalitäten der Beweiserhebung im Rahmen von Untersuchungsverfahren auseinander. Beispielsweise hatte der Staatsgerichtshof Bremen sich mit dem Umfang des Aktenvorlagerechts zu befassen ${ }^{65}$, das den Untersuchungsausschüssen in vielen Landesverfassungen schon vor Einführung des oben beschriebenen allgemeinen Aktenvorlagerechts zugestanden wurde. Der Staatsgerichtshof stellte fest, dass die Behörden und Dienststellen des Landes grundsätzlich verpflichtet sind, die geforderten Akten herauszugeben, auch wenn die allgemeinen Verfahrensvorschriften ${ }^{66}$ unter bestimmen Voraussetzungen zur Ablehnung eines entsprechenden Gesuchs berechtigen. Zur Begründung wurde ausgeführt, dass den Behörden, deren Tätigkeit im Rahmen des Untersuchungsverfahrens kontrolliert werde, kein „Gegenrecht" ${ }^{“ 67}$ eingeräumt werden dürfe, durch das der Untersuchungszweck vereitelt werden könne. Die Grenzen des Aktenvorlageanspruchs seien direkt der Landesverfassung zu entnehmen. ${ }^{68}$ So müsse der Grundsatz der Verhältnismäßigkeit gewahrt und darauf geachtet werden, dass insbesondere die Vorlage von Personalakten mit Grundrechten kollidieren könne. ${ }^{69}$

Obwohl die Landesverfassungsgerichte zahlreiche Entscheidungen zu Rechten und Pflichten von Parlamentarischen Untersuchungsausschüssen getroffen haben, erscheint ein direkter Einfluss auf die Positivierung in Form von Gesetzen über das Verfahren von Untersuchungsausschüssen als eher unwahrscheinlich. Wie aus Tabelle 1 ersichtlich ist, übernahmen die Länder Bayern (1970), Berlin (1970) und Baden-Württemberg (1976) beim Erlass

60 Vgl. Entscheidung des StGH Baden-Württemberg vom 16. April 1977, ESVGH 27, S. 1.

61 Vgl. Entscheidungen des StGH Hessen vom 24. November 1966 und 9. Februar 1972, ESVGH 17, S. 1 und ESVGH 22, S. 136.

62 Vgl. Entscheidung des VerfGH Bayern vom 27. Juni 1977, BayVerfGHE 30, S. 48.

63 ESVGH 17, S. 1 (S. 10).

64 Vgl. Entscheidung des VerfGH Bayern vom 27. Juni 1977, BayVerfGHE 30, S. 48.

65 Vgl. Entscheidung vom 13. März 1978, BremStGHE 1977/1979, 75.

66 Vgl. zum Beispiel $\$ 96$ StPO.

67 BremStGHE 1977/1979, 75 (S. 94).

68 Vgl. ebenda, S. 93.

69 Vgl. ebenda, S. 94. 
entsprechender gesetzlicher Vorschriften eine Vorreiterrolle. Die verfassungsgerichtliche Rechtsprechung hierzu setzte in Baden-Württemberg und Bayern aber erst im Jahr 1977 ein. In Berlin, dessen Verfassungsgericht ohnehin erst 1992 seine Arbeit aufnahm, sind bis Ende 2007 keine Organstreitverfahren zu diesem Themenkomplex entschieden worden. Dagegen hat die Rechtsprechung der Staatsgerichtshöfe in $\operatorname{Hessen}^{70}$ und Niedersachsen ${ }^{71}$ bislang nicht zur gesetzlichen Regelung des Untersuchungsausschussverfahrens geführt, wohingegen die neuen Bundesländer in der Mehrzahl entsprechende Gesetze bereits vor der Konstituierung ihrer Verfassungsgerichte erlassen haben (so Brandenburg, Sachsen, Sachsen-Anhalt und Thüringen).

Einzig in Bremen ist aufgrund der zeitlichen Reihenfolge ein Einfluss der Rechtsprechung des Staatsgerichtshofs auf die Positivierung des Untersuchungsausschussrechts nicht ausgeschlossen. Zwei Entscheidungen waren hierzu bereits ergangen ${ }^{72}$, als das Untersuchungsausschussgesetz im Jahr 1982 verabschiedet wurde. Allerdings war die Bremische Bürgerschaft schon vor den Entscheidungen des Staatsgerichtshofs mit der Beratung von gesetzlichen Änderungen im Bereich des Untersuchungsausschusswesens befasst. Den Grund hierfür lieferte die einschlägige Vorschrift der Landesverfassung (Art. 105 Abs. 6 a.F. LV Bremen), die die Einsetzung Parlamentarischer Untersuchungsausschüsse mit ungewöhnlich hohen Verfahrensvoraussetzungen verband. Im Gegensatz zu fast allen anderen Landesverfassungen war für die Einsetzung eine parlamentarische Mehrheit notwendig, während in den anderen Bundesländern schon damals der Antrag einer qualifizierten Minderheit ausreichend war. ${ }^{73}$ Die Nutzung des Kontrollinstruments war der Opposition daher stark erschwert, weshalb es schon 1968 und 1970 Initiativen gab, die eine Änderung des Art. 105 Abs. 6 LV Bremen zum Ziel hatten. ${ }^{74}$ Etwa zeitgleich begannen die Beratungen von Entwürfen zum Erlass eines Untersuchungsausschussgesetzes. Den Anlass bildete ein Untersuchungsverfahren 1969/1970, das Grundstücksgeschäfte in Bremen zum Inhalt hatte. ${ }^{75}$ Nach wiederholten Initiativen brachte die CDU-Fraktion 1980 einen Gesetzentwurf ein, der nach zwei Jahren Beratung schließlich verabschiedet wurde. ${ }^{76}$

Die Rechtsprechung des Staatsgerichtshofs zu Untersuchungsausschüssen spielte bei den Gesetzesberatungen offenbar nur eine untergeordnete Rolle. Bei der Formulierung des Gesetzentwurfs orientierte man sich vor allem an Vorschlägen der Interparlamentarischen Arbeitsgemeinschaft und an Empfehlungen der Landtagspräsidentenkonferenz. ${ }^{77}$ Während

70 Vgl. Entscheidungen vom 24. November 1966 und 9. Februar 1972, ESVGH 17, S. 1 und ESVGH 22, S. 136.

71 Vgl. Entscheidungen vom 16. Januar 1986, NdsStGHE 3, S. 1, S. 8.

72 Vgl. Entscheidungen vom 17. April 1970 und 13. März 1978, BremStGHE 1970/1976, 11 und BremStGHE 1977/1979, 75.

73 Untersuchungsausschüsse können heute in allen Bundesländern auf Antrag von einem Viertel beziehungsweise einem Fünftel der Abgeordneten eingerichtet werden. Im Saarland war bis 1979 der Antrag von einem Drittel der Abgeordneten erforderlich (Art. 81 Abs. 1 LV Saarland). Nur Berlin sah bis 1970 ebenso wie Bremen einen Mehrheitsbeschluss vor (Art. 33 Abs. 1 a.F. LV Berlin).

74 Vgl. Abgeordneter Johann-Tönjes Cassens (CDU), Plenarprotokoll Nr. 8/78 vom 19. Juni 1975, S. $4372 \mathrm{D}$.

75 Vgl. Abgeordneter Hermann Ehlers (CDU), Plenarprotokoll 10/67 vom 29. September 1982, S. 5195 D.

76 Vgl. Drucksache 10/306.

77 Vgl. Hermann Ehlers a.a.O. (Fn. 75). 
der Beratungen wurden die Entscheidungen des Staatsgerichtshofs nur am Rande erwähnt. ${ }^{78}$ Die Rolle eines Ersatzgesetzgebers hat der Bremische Staatsgerichtshof in dieser Frage demnach nicht übernommen.

\section{Fazit: Vielfältige Einflüsse auf die Fortentwicklung des Parlamentsrechts}

Zusammenfassend kann festgehalten werden, dass die Landesverfassungsgerichte zwar zahlreiche Entscheidungen zu Fragen des Parlamentsrechts getroffen haben, aber kaum als Ersatzverfassungs- beziehungsweise Ersatzgesetzgeber qualifiziert werden können. Die Aufnahme von Minderheitsrechten in die Landesverfassungen beziehungsweise die gesetzlichen Regelungen zu den Verfahren der Untersuchungsausschüsse lassen sich nicht auf die verfassungsgerichtliche Rechtsprechung zurückführen. Besonders deutlich wird dies in Bezug auf die Ausgestaltung des Zitierrechts als Minderheitsanspruch und die Berichtspflichten der Regierung. Zu beiden Bereichen lassen sich keine verfassungsgerichtlichen Entscheidungen finden, weder vor noch nach der Normierung der Rechte in einzelnen Landesverfassungen. Die selbständigen Auskunftsrechte und die Ansprüche auf die Vorlage von Regierungsakten wurden erst zum Gegenstand von Organstreitverfahren, nachdem entsprechende Regelungen in die Landesverfassungen aufgenommen worden waren. Hierbei ist jedoch eine wichtige Ausnahme zu erwähnen: Der Nordrhein-Westfälische Verfassungsgerichtshof hat die Existenz von selbständigen Auskunftsrechten der Abgeordneten grundsätzlich bejaht ${ }^{79}$, ohne dass die Landesverfassung eine entsprechende Vorschrift enthält. Diese Entscheidung kann in der Tat als ein Beispiel für gerichtliche Verfassungsgebung gewertet werden.

Hinsichtlich der Festschreibung des Oppositionsstatus in den meisten Landesverfassungen dürfte die landesverfassungsgerichtliche Rechtsprechung keinen Einfluss gehabt haben. Die richterliche Auseinandersetzung hiermit erfolgte erst, nachdem in Hamburg bereits eine entsprechende Verfassungsregelung verabschiedet worden war. Ein gewisser Einfluss der Landesverfassungsgerichte auf das Parlamentsrecht lässt sich allerdings in Bezug auf die Stellung der Fraktionen feststellen. So sprach der Staatsgerichtshof Bremen den Fraktionen den Rang von ständigen, mit eigenen Rechten ausgestatteten Gliederungen der Bürgerschaft zu, weshalb insbesondere die Fraktionsbildung zu den Aufgaben der Fraktion gehörte, die der Einwirkung der Partei entzogen seien. ${ }^{80}$ Zwei Entscheidungen des Bayerischen Verfassungsgerichtshofs stärkten die Position der Fraktionen, indem ihnen der Rang von mit eigenen Rechten ausgestatteten Teilen eines Staatsorgans zugeschrieben wurde. ${ }^{81} \mathrm{Ob}$ wohl die Bayerische Landesverfassung nach ihrem Wortlaut keine Antragsberechtigung von Fraktionen in Organstreitverfahren vorsah, wurde diese durch die Rechtsprechung des Verfassungsgerichtshofs eingeführt. Für die spätere Positivierung der Fraktionsrechte in den Verfassungen von Bremen und Bayern spielten die genannten Entscheidungen aber offenbar keine Rolle. Weder in den schriftlichen Begründungen der entsprechenden Gesetzentwürfe noch in den parlamentarischen Lesungen wurde auf die Judikate der Landesverfassungsgerichte Bezug genommen.

78 Vgl. Abgeordneter Claus Jäger (FDP), Plenarprotokoll 10/67 vom 29. September 1982, S. 5199 C.

79 Vgl. Urteil vom 4. Oktober 1993, OVGE MüLü 43, S. 274.

80 Vgl. BremStGHE 1970/1976, 19.

81 Vgl. BayVerfGHE 25, S. 97; BayVerfGHE 29, S. 62. 
Von großer Bedeutung für die Entwicklung des Parlamentsrechts ist dagegen die Rechtsprechung der Landesverfassungsgerichte zu Untersuchungsausschüssen. Hierzu sind zahlreiche Entscheidungen ergangen, die die gesamte Bandbreite des Themenkomplexes abdecken. So haben sich die Gerichte zu den Modalitäten der Einsetzung und der Beweisaufnahme, zu Minderheitsrechten und Statusfragen geäußert. Ein direkter Einfluss auf die Positivierung des Rechts der Untersuchungsausschüsse ist allerdings nicht erkennbar. So setzte in mehreren Ländern die gerichtliche Auseinandersetzung mit dem Untersuchungsausschussrecht erst nach Erlass entsprechender gesetzlicher Vorschriften ein (zum Beispiel in Baden-Württemberg und Bayern). In anderen Ländern waren die Gerichte nie mit der Klärung entsprechender Fragen befasst (zum Beispiel in Berlin und Rheinland-Pfalz). Eine weitere Ländergruppe, bestehend aus Hessen, Niedersachsen und dem Saarland, hat bislang auf die gesetzliche Regelung von parlamentarischen Untersuchungsverfahren verzichtet. Es kann davon ausgegangen werden, dass die Staatsgerichtshöfe von Hessen und Niedersachsen, die jeweils mehrere einschlägige Entscheidungen getroffen haben, aufgrund des Fehlens entsprechender gesetzlicher Vorschriften am ehesten als „Ersatzgesetzgeber" qualifiziert werden können. Insgesamt spricht aber wenig dafür, dass es sich bei der Abfolge von „Konflikt Organstreit - Entscheidung - Aufnahme ins geschriebene Recht" um einen "typischen Entwicklungspfad" ${ }^{82}$ des Parlamentsrechts handelt.

Damit stellt sich die Frage, welche anderen Institutionen die Ausweitung von Minderheitsrechten im Parlamentsrecht der Bundesländer gefördert haben. Hier ist zum einen das Bundesverfassungsgericht zu nennen. Obwohl die Rechtsräume von Landes- und Bundesebene grundsätzlich eigenständig sind, wurde das Parlamentsrecht der Länder auch durch die bundesverfassungsgerichtliche Rechtsprechung geprägt. Dies gilt beispielsweise für die Klärung des Status von Fraktionen. Der Begriff wird schon in den frühen Entscheidungen des Bundesverfassungsgerichts verwendet und umschrieben. Dabei werden Fraktionen in der Regel als „ständige Gliederungen“ des Parlaments bezeichnet, die mit eigenen Rechten ausgestattet sind und denen die Aufgabe zukommt, „den technischen Ablauf der Parla-

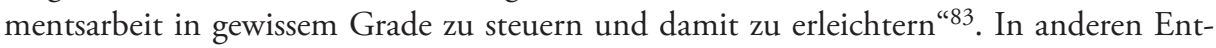
scheidungen bezeichnet das Gericht Fraktionen als „von der Verfassung anerkannte Teile eines Verfassungsorgans" 84 oder als „notwendige Einrichtungen des Verfassungslebens" ${ }^{\text {" }}$. Ähnliche Umschreibungen enthalten die in den 1990er Jahren in mehrere Landesverfassungen aufgenommenen Vorschriften. Dass diese Übereinstimmungen beabsichtigt sind, lässt sich beispielsweise anhand der parlamentarischen Materialien zur Schaffung der neuen Verfassungsregelungen belegen. Hier wird zum Teil ausdrücklich auf die Rechtsprechung des Bundesverfassungsgerichts verwiesen, so zum Beispiel in Bremen. ${ }^{86}$

Ähnliches gilt für die Schaffung des bremischen Untersuchungsausschussgesetzes. Zur Frage der Erweiterung des Untersuchungsgegenstands durch die parlamentarische Mehrheit wurde auf eine Entscheidung des Bundesverfassungsgerichts verwiesen, die es in seiner

82 Pascale Cancik, a.a.O. (Fn. 11), S. 584 (Hervorhebung im Original).

83 BVerfGE 1, S. 208 (229); S. 351 (S. 359); BVerfGE 2, S. 143 (S. 160, S. 167); S. 347 (S. 365); BVerfGE 10, S. 4 (S. 14).

84 BVerfGE 43, S. 142 (S. 147).

85 BVerfGE 70, S. 324 (S. 362). Vgl. Hanno Pfeil, Der Abgeordnete und die Fraktion - verfassungsrechtliche Vorgaben und gesetzliche sowie binnenrechtliche Ausgestaltung, Hamburg 2008, S. 67.

86 Vgl. Drucksache 13/592 vom 21. Juni 1993. 
Eigenschaft als Landesverfassungsgericht von Schleswig-Holstein getroffen hatte. ${ }^{87}$ Hinzu kommt, dass sich die Verfassungsgerichte der Länder häufig an der Rechtsprechung des Bundesverfassungsgerichts orientieren. Dies gilt selbst dann, wenn die normativen Grundlagen von Grundgesetz und Landesverfassung nicht miteinander übereinstimmen. ${ }^{88}$ Die bundesverfassungsgerichtliche Rechtsprechung beeinflusst also sowohl die parlamentarische Beratung von Gesetzentwürfen als auch die Entscheidungen der Landesverfassungsgerichte.

Noch bedeutender als die Rechtsprechung des Bundesverfassungsgerichts dürfte für die Erweiterung der parlamentarischen Minderheitsrechte die Orientierung an Verfassungsvorschriften anderer Bundesländer gewesen sein. So diente der Oppositionsartikel der Verfassung von Hamburg (Art. 24) als Vorbild für eine entsprechende Regelung in der Landesverfassung von Schleswig-Holstein. ${ }^{89}$ Die Aufnahme der Regelung war dabei Teil einer umfassenden Verfassungs- und Parlamentsreform, die als Reaktion auf die so genannte Barschel-Affäre in den späten 1980er Jahren initiiert wurde und die letztlich zur Ablösung der bis dahin geltenden Landesssatzung durch die neue Landesverfassung im Jahr 1990 führte. Wie sich aus Tabelle 1 entnehmen lässt, wurde zum damaligen Zeitpunkt nicht nur der Oppositionsstatus verfassungsrechtlich verankert, sondern es wurden auch die Rechte der parlamentarischen Minderheit umfassend ausgebaut. So gestaltete Schleswig-Holstein als erstes Bundesland das Zitierrecht als Minderheitsanspruch aus und nahm bei der Normierung der Auskunftsrechte einzelner Abgeordneter sowie der Berichtspflichten der Landesregierung eine Vorreiterrolle ein.

Die schleswig-holsteinischen Bestimmungen zum Parlamentsrecht dienten wiederum als Vorbild für die in den neuen Bundesländern zu schaffenden Verfassungen, wurden aber zum Teil auch in einzelne westdeutsche Verfassungen integriert. ${ }^{90}$ Beispielsweise wurden die selbständigen Auskunftsrechte der Abgeordneten in den Verfassungen der neuen Bundesländer der schleswig-holsteinischen Regelung nachgebildet ${ }^{91}$, ebenso die Berichtspflichten der Landesregierungen. ${ }^{92}$ Für die Verbreitung der schleswig-holsteinischen Regelungen dürfte auch eine entsprechende Empfehlung der Landtagspräsidentenkonferenz beigetragen haben. ${ }^{93}$ Im Jahr 1991 beauftragte dieses Gremium eine Kommission der Landtagsdirekto-

87 Vgl. Abgeordneter Axel Adamietz (BGL), Plenarprotokoll 10/70 vom 4. November 1982, S. 5428D.

88 Vgl. Otto Bachof, Diskussionsbeitrag, in: Veröffentlichungen der Vereinigung der deutschen Staatsrechtslehrer, 46. Jg. (1988), S. 141 - 143, S. 141; Bodo Pieroth, Organstreitverfahren vor dem Verfassungsgerichtshof, in: Verfassungsgerichtsbarkeit in Nordrhein-Westfalen. Festschrift zum 50-jährigen Bestehen des Verfassungsgerichtshofs für das Land Nordrhein-Westfalen, Stuttgart 2002, S. $103-116$, S. 115.

89 Vgl. Schleswig-Holsteinischer Landtag (Hrsg.), Schlussbericht der Enquete-Kommission Verfassungs- und Parlamentsreform, Baden-Baden 1989, S. 44.

$90 \mathrm{Zu}$ den Nachweisen für die Vorbildwirkung der schleswig-holsteinischen Regelungen in Sachsen und Brandenburg vgl. Pascale Cancik, Parlamentarische Opposition in den Landesverfassungen. Eine verfassungsrechtliche Analyse der neuen Oppositionsregelungen, Berlin 2000, S. 82.

91 Vgl. Andrea Stiens, Chancen und Grenzen der Landesverfassungen im deutschen Bundesstaat der Gegenwart, Berlin 1997, S. 106.

92 Vgl. Matthias Niedobitek, Neuere Entwicklungen im Verfassungsrecht der deutschen Länder, Speyer 1994, S. 32.

93 Vgl. Konferenz der Landtagspräsidenten, Empfehlung der Präsidentinnen und Präsidenten der deutschen Landesparlamente zum Informationsrecht des Abgeordneten und des Parlamentes sowie zu den Informationspflichten der Regierung, in: ZParl, 23. Jg. (1992), H. 4, S. 573 - 582. 
ren mit der „Erarbeitung einer Vorlage zur Frage der Zweckmäßigkeit und zum möglichen Inhalt eines Parlamentsinformationsgesetzes sowie eines Entwurfes entsprechender Vorschriften "94. Den Auslöser für die Einsetzung der Kommission bildete ein Bericht der Präsidentin des Landtags von Schleswig-Holstein über die dortige Verfassungs- und Parlamentsreform. In ihrem Bericht empfahl die Kommission die Ergänzung der Landesverfassungen um selbständige Auskunftsrechte sowie um Aktenvorlageansprüche zugunsten der Landtagsausschüsse. Zudem wurde angeregt, die Unterrichtungsverpflichtung der Landesregierung zu übernehmen und die Details im Rahmen eines Parlamentsinformationsgesetzes zu regeln. ${ }^{95}$ Obwohl der genaue Wortlaut der empfohlenen Verfassungsvorschriften nicht mit jenem der schleswig-holsteinischen Regelungen übereinstimmt, ist deren Vorbildcharakter deutlich zu erkennen.

Auch die Aktenvorlageansprüche in den Landesverfassungen von Niedersachsen und Sachsen-Anhalt folgen dem schleswig-holsteinischen Vorbild. Die Vorschriften in den drei genannten Ländern zeichnen sich dadurch aus, dass sie den Landesregierungen das Recht einräumen, die Aktenvorlage zu verweigern, wenn ihre Funktionsfähigkeit und Eigenverantwortung hierdurch beeinträchtigt wird. ${ }^{96}$ Diese Formulierung ist wiederum durch das Urteil des Bundesverfassungsgerichts zum Flick-Untersuchungsausschuss beeinflusst. ${ }^{97}$ Hierbei ging es um die Frage, ob die Weigerung der Bundesregierung, dem Untersuchungsausschuss bestimmte Akten vorzulegen, einen Verstoß gegen Art. 44 GG darstelle. Das Bundesverfassungsgericht urteilte in diesem Zusammenhang, dass es zur Sicherung der Gewaltenteilung erforderlich sei, der Regierung einen „Kernbereich exekutiver Eigenverantwortung“ zuzugestehen, der einen „grundsätzlich nicht ausforschbaren Initiativ-, Beratungs- und Handlungsbereich einschließt ${ }^{\text {" } 98}$. Die Aktenvorlageansprüche in Niedersachsen, Schleswig-Holstein und Sachsen-Anhalt sind daher sowohl auf Vorbilder in anderen Landesverfassungen als auch auf die Einbeziehung der bundesverfassungsgerichtlichen Rechtsprechung zurückzuführen..$^{99}$

Es sind demnach vielfältige Einflüsse, die zur Fortentwicklung des Parlamentsrechts in den Bundesländern beigetragen haben. Von besonderer Bedeutung ist dabei die Orientierung an Vorbildern in anderen Landesverfassungen. Auch die Rechtsprechung des Bundesverfassungsgerichts wird in den Bundesländern rezipiert. Der Anteil der Parlamentsrechtsänderungen, der auf den Einfluss der Landesverfassungsgerichte zurückgeführt werden kann, ist dagegen trotz der Fülle der vorhandenen Rechtsprechung als gering zu bewerten.

94 Ebenda, S. 573.

95 Vgl. ebenda, S. $574 \mathrm{f}$.

96 Vgl. Art. 24 Abs. 3 LV Niedersachsen; Art. 53 Abs. 4 LV Sachsen-Anhalt; Art. 23 Abs. 3 LV Schleswig-Holstein.

97 Vgl. BVerfGE 67, S. 100.

98 Ebenda, S. 139.

99 Vgl. Entscheidung vom 15. Mai 1996, NdsStGHE 3, S. 252 (S. 255 f.). 